\title{
Kronenverlichtung und Sterberaten bei Fichten, Tannen und Buchen
}

\author{
Von P. Schmo-Hans
}

\section{Die Bedeutung eines Merkmals für Diagnose und Prognose}

Der Gesundheitszustand eines Baumes muß im wesentlichen auf Grund von Größe, Strukiur, Farbe und Dichte seiner Krone beurteilt werden; in Ausnahmefallen sind Symptome an Stamm oder Wurzeln zu erkennen. Nadel-/Blattverluste und Vergilbungen sind die in den letzten Jahren am genauesten untersuchten einzelnen Merkmale.

Leider sind Nadel-/Blattverluste ein ziemlich unspezifisches Phänomen; sie lassen sich nicht klar auf eine Ursache zurückführen, ihr diagnostischer Wert wird daher häufig bezweifelt. Anderseits sind Nadel- und Blattverluste quantitativ und relativ objektiv ansprechbar. Die meisten der vorgeschlagenen Kronenstrukturmerkmale (Lesisski u. LANDMAN 1990) bereiten in wiederholten Waldinventuren jedenfalls größere Schwierigkeiren. Der diagnostische Wert eines Merkmals wird erst definitiv beurteilt werden können, wenn die Ursachen oder physiologischen Zusammmenhänge einer Schädigung erkannt sind.

Ebenso wichtig wie die Abklärung von Art und Ursache einer Schädigung ist die Kenntnis des prognostischen Wertes eines Merkmals, sollte doch nicht nur die Art, sondern auch der Schweregrad einer Schädigung erkannt werden. Ein Phänomen ist nicht immer gravierend, wenn der Baum momentan schlimm aussieht, sondern nur, wenn die Prognose für einen so aussehenden Baum schlecht ist. Nur die Kenntnis der nachfolgenden Entwicklung von Bäumen mit einer bestimmten Merkmalskombination läßt beurteilen, wie schwerwiegend eine Schadigung ist.

Eine naheliegende Beurteilung des prognostischen Wertes eines Merkmals ertolgr auf Grund der weiteren Entwicklung dieses Merkmals selbst. Wenn sich ein Merkmal im allgemeinen nach kurzer Zeit wieder zurückbildet, ist es ein weniger schlimmes Zeichen, als wenn häufig mit seiner immer stärkeren Ausprägung gerechnet werden muß.

Besonders wichtige Indizien für den Gesundheitszustand eines Baumes sind Größe und Verlauf des Zuwachses, selbstverständlich auch dann, wenn die Holzprodulstion nicht primäres Ziel ist. Zwar ist ein kurefristig großer Zuwachs oder eine Zuwachsbeschleunigung nicht immer Zeichen guter Gesundheit oder Stabilität; eine über mehrere Jahre anhaltende markante Zuwachsverlangsamung ist aber untrügliches Zeichen einer Schädigung oder einer markanten Verschlechterung der Umweltbedingungen dieses Baumes oder Bestandes.

Ein noch eindeutigeres Maß für den Grad einer Schädigung ist die Überlebenswahrscheinlichkeit eines Baumes. Wenn Bäume mit einem bestimmten Merkmal häufiger absterben als andere, hat dieses Merkmal einen wichtigen prognostischen Wert. 


\section{Kronenverteilung und Zwangsnutzung ohne starken Sturm}

In einem Teil der ertragkundlichen, regelmäßig gepflegten Versuchsflächen wurden zwischen 1985 und 1991 jährlich oder in Abständen von höchstens 4 Jahren alle Kronen angesprochen (KeLLER u. IMHOF 1987; KeLLER u. STARK 1991); gleichzeitig wurde notiert, welche Bäume überlebr hatten, welche und aus welchem Grund zwangsweise genutzt worden oder abgestorben waren. Alle Ergebnisse sind von W. KELLER zur Verfügung gestellt worden.

Trotz der guten Kontrolle dieser Flächen und der im allgemeinen sehr guten Zusammenarbeit mit den lokalen Forstdiensten konnte nicht immer ausgeschlossen werden, daß bei $Z$ wangsnurzungen nicht auch noch Bäume aus waldbaulichen Gründen gefällt worden waren. Diejenigen Flächen, in denen derartige Nutzungen nicht mit einiger Sicherheit ausgeschlossen werden konnten, wurden nicht in diese Untersuchung aufgenommen, ebonsowenig Flächen, in denen die Bälime ielweise infolge zu starker Konkurrenz abgestorben sind. Um ganz sicher zu gehen, wurden auch alle Flächen ausgeschieden, in denen in den Beobachtungsjahren Durchforstungen oder Plenterungen durchgeführt worden waren, weil unter Umständen nicht immer zwischen waldbaulich bedingten und erzwungenen Eingriffen unterschieden werden konnte und weil eventuell durch Eingriffe bedingte Folgeschäden härten eintreten können.

In den verbleibenden Flächen wurden, um die Daten nicht zu verfälschen, alle Bäume berücksichtigr. Diese sind aus den verschiedensten Gründen (lokaler Sturm, Stockfäule, Hallimaschbefall, Insekten, Blitz etc.) ausgefallen, manchmal konnten mehrere Ursachen gleichzeitig festgestell werden, häufig blieb die Ursache unbekannt. Die Auswertung wurde nur für die Gesamtheir aller Zwangsnutzungen und nicht für die einzelnen Ursachen getrennt durchgeführt, weil die Häufigkeiten für die einzelnen bekannten Ursachen zu klein waren. Leider sind einige Bäume, die bei der Ernte von spontan abgestorbenen Nachbarn verletzt und deshaib ebenfalls genutze worden waren, in den Häufigkeiten der zwangsweise Entnommenen enthalten.

In 10 von KELLER und IMHOF (1987) beschriebenen Plenterflächen konnte das Überleben oder Absterben von 1652 Fichten, 1157 Tannen und 35 Buchen während bis zu sechs Jahren von 1985 bis 1991 beobachter werden. Insgesamt wurden Fichten in 5622, Tannen in 5083 und Buchen in 174 Jahren beobachtet. In 2 gleichaltrigen Fichten-, 7 Buchen- und 13 gemischten Versuchsflächen wurden während der gleichen Zeit 1207 Fichten, 493 Tannen und 1123 Buchen beobachtet; insgesamt Fichten in 3517, Tannen in 1977 und Buchen in 3290 Jahren. Die Flächen befinden sich zu einem großen Teil im Mittelland und in den Voralpen, teilweise aber auch im Jura und in den Alpen. Sie sind verschieden stark und seit Jahrzehnten in einem regelmäßigen Turnus durchforstet worden.

In den untersuchen Flächen sind pro Jahr durchschnittlich $0,5 \%$ der Fichten, 1,3\% der Tannen und $0,8 \%$ der Buchen abgestorben oder erzwungenermaßen genutzt worden. Die Ausfallrate ist bei Fichten, Tannen und Buchen stark abhängig von den Nadel-/ Blattverlusten (Abb. 1). Die empirisch ermittelten relativen Häufigkeiten lassen sich gut durch eine logistische Funktion ausgleichen. Die Ausfallrate nimmt bei kleinen NadelverLusten nur langsam, dann aber immer rascher zu; der statistische Zusammenhang $z$ wischen Nadelverlust und Ausfallrate ist hoch signifikant $(p<0,001)$.

In Dauerversuchsflächen in Österreich errechnen sich aus den stehend tot aufgefundenen Bäumen allein Mortalitätsraten von $0,01 \%, 0,05 \%, 0,34 \%$ und $4,03 \%$ für die österreichischen Kronenverlichtungsstufen 1 bis 4. Die Mortalitätsraten verhalten sich somit wie 1:5:30:400 für die Gesamtheit aller beobachteten Baumarten (STEMBERger 1991). Die in der Schweiz festgestellten Relationen, 1:2:7:300 für Fichten, 1:2:5:60 für Tannen und 1:1:3:60 für Buchen können in Anbetracht der methodischen Unterschiede weitgehend als Bestärigung gelten. 


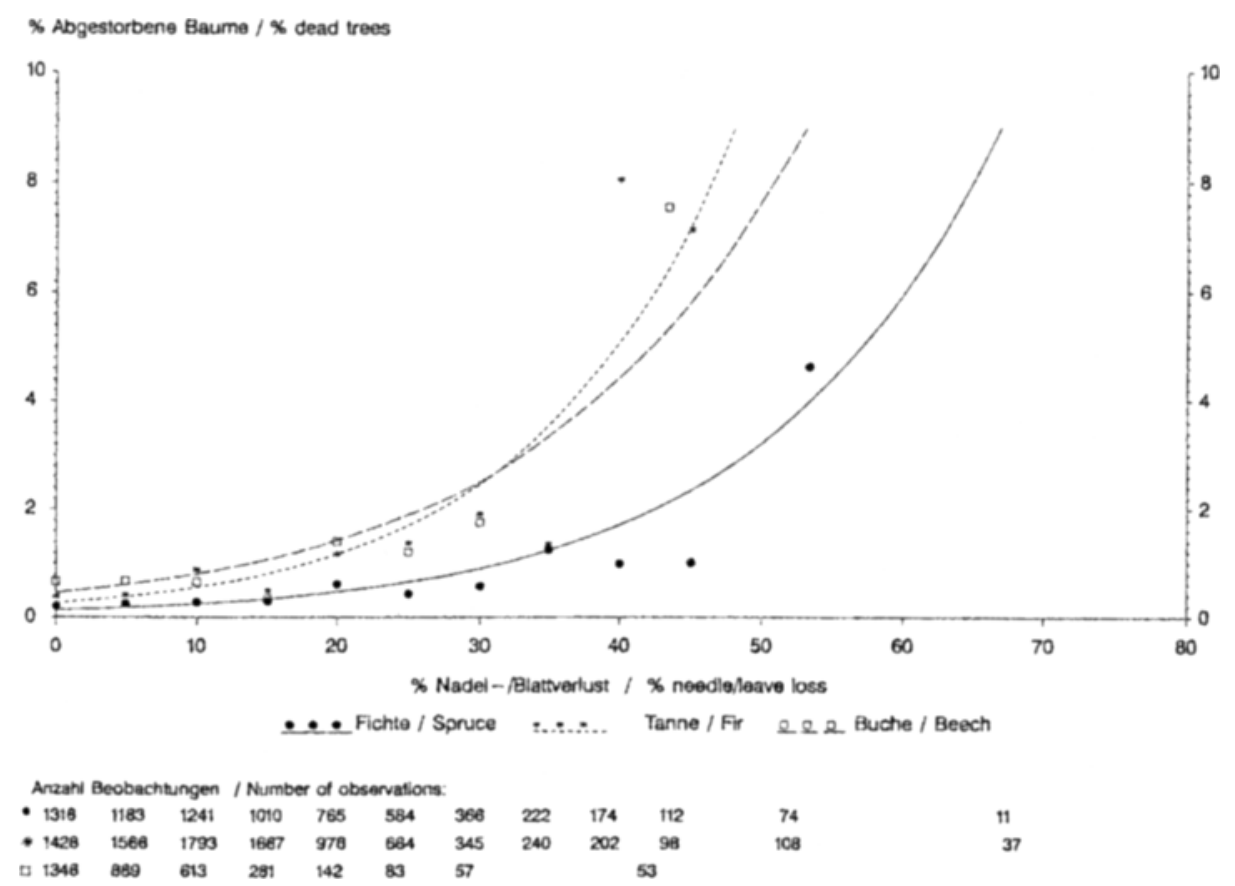

Abb. 1. Zwangsnutzungen in Plenter- und gleichaltrigen Hochwäldern in Abhängigkeit vom Nadel-1 Blattverlust. Ausgleichstunktionen durch gewichtete logistische Regressionen berechnet

Fig. 1. Salvage cuttings in even-aged and uneven-aged forests in relation to needie or leave loss. Smoothing functions were calculated by logistic regressions

Innerhalb der Bestände wurde mi multipler logistischer Regression (SAS Programm) neben Baumart und Nadel-/Blattverlust kein weiteres Merkmal gefunden, das einen signifikanten zusätzlichen Beitrag zur Schätzung der Sterbewahrscheinlichkeit liefert. Weder im Hochwald noch im Plenterwald gibt die soziale Stellung eines Baumes eine zusätzliche Information darüber. Auch der Durchmesser in Brusthöhe zeigt keinen zusätzlichen statistischen Zusammenhang mit der jährlichen Ausfalliate, weder für Fichte noch für Tanne oder Buche, weder im Plenterwald noch im gleichaltrigen Hochwald.

Die Ausfallraten sind in einzelnen Beobachtungsjahren etwas höher als in anderen ausgefallen, doch die Aufzeichnungen genügen bei weitem nicht, um die Unterschiede auf bestimmte Witterungsmerkmale zurückzuführen. Auch zwischen den Beobachtungsflächen bestehen große Unterschiede in der Höhe der erzwungenen Nutzungen. In mehreren Flächen mußte in der Beobachtungsperiode kein einziger Baum zwangsweise entnommen werden. Diese Unterschiede können aber, vielleicht weil zu wenig Flächen untersucht werden konnten, nicht auf eines oder mehrere der erhobenen Standortsmerkmale zurückgeführt werden. Sie sind auch nicht eindeutig von den pflanzensoziologischen Einheiten abhängig. Zwischen Plenterwald und gleichaltrigem Hochwald besteht bei gleichen Nadelverlusten ebenfalis kein signifikanter Unterschied. Bei Buchen konnte zusätzlich der Einfluß der Durchforstungstätigkeit untersucht werden; die Ausfallraten unterscheiden sich nicht signifikant, weder bei Hoch- und Niederdurchforstung noch bei verschiedenen Durchforstungsstärken. Allerdings sterben mitherrschende und beherrschre Buchen in mäßig durchforsteten Beständen natürlich etwas häufiger ab.

In den gleichaltrigen Beständen nimmt der Nadel-/Blattverlust mit dem Alter nicht zu; bei gegebener Kronenverlichtung ist die Sterberate nicht einmal tendenziell mit dem Alter 
steigend, obwohl die Bestandesalter der untersuchten Flächen im Bereich zwischen 80 und 210 Jahren streuen. Auch dieses erstaunliche Resultat bestätigt nur, was in Österreich bereits festgestellt worden ist (STEMBERGER 1991).

\section{Kronenverlichtung und Sturmgefährdung}

Im Forstbetrieb Lenzburg konnte statistisch einwandfrei nachgewiesen werden, daß Abteilungen, in denen die Kronen auf den Infrarot-Luftbildern als stärker verlichtet eingestuft worden waren, durch die außerordentlichen Winterstürme 1990 entgegen den Erwartungen durchschnitrlich größere Schäden (Verhältnis von Zwangsnutzungsmenge zu Vorrat) erlitten als Abteilungen mit besser aussehenden Kronen (LäII 1991). Im Forstbetrieb Zofingen wurde dieser Zusammenhang für Bestände, die auf dem Luftbild als verschieden stark geschädigt angesprochen worden waren, bestätigt (Daten E. STEINER 1991).

In Oberentfelden waren 1985 die Kronen aller vorherrschenden, herrschenden und mitherrschenden Bäume in 274 Kontroll-Stichprobeflächen von je $500 \mathrm{~m}^{2}$ terrestrisch auf ihren Nadel-/Blattverlust angesprochen worden (Frscher u. Rerter 1985). Die Stürme haben im Winter 1990 einen großen Teil dieses Waldes geschädigt, teilweise in der Form kleiner Flächenwürfe, vor allem aber durch einzelbaumweise Streuschäden. Bei jedem Baum in den Probetlächen mit mehr als 50 Jahre alten Beständen wurde festgestellt, ob er schon vor den Stürmen genutzt, durch die Stürme gefüllt oder stehengeblieben war. Insgesamt sind durch die Stürme $12 \%$ der beobachteten Fichten, $13 \%$ der Tannen, $2 \%$ der Buchen und $3 \%$ der übrigen Baumarten geschädigt worden. Leider konnte nicht mehr festgestellt werden, ob es sich jeweils um einen Stammbruch, Stockbruch, Wurzelbruch oder Wurf gehandelt hatte.

Die statistische Auswertung mit multiplen logistischen Regressionen (SAS-Programm) hat ergeben, daß die Sturmgefährdung eng mit den Nadelverlusten von Fichten und Tannen korrelicrt ist (SCFMID-HAAs 1991). Aus Abbildung 2 ist ersichtlich, wie stark die Sturmgefährdung von Fichten und Tannen schon bei sehr kleinen Nadelverlusten zunimmt. Fichten mit 15 bis $25 \%$ Nadelverlust sind ungefähr 1,6 mal, Tannen sogar 2,3 mal so häufig von den Stürmen gebrochen oder geworfen worden wie diejenigen mit $O$ bis $10 \%$ Nadelverlust. Der Unterschied zwischen den beiden Kronenverlichtungsstufen ist für beide Baumarten nach dem Chi-Quadrat-Test statistisch signifikant ( $\mathrm{p}<0,05$ für Fichte und $\mathrm{p}<0,01$ für Tanne). Da nur 2 Fichten und 20 Tannen mehr als $25 \%$ Nadelverlust aufgewiesen hatten, kann die stärkere Gefährdung dieser Bäume nicht mit genügender Sicherheit belegt werden. Anderseits sind sogar die Unterschiede innerhalb der ersten Stufe, also zwischen 0 und $10 \%$ Nadelverlust, sowohl für Fichte als auch für Tanne statistisch signifikant $(p<0,01)$. Keine einzige der 90 Tannen mit einem Nadelverlust von $0 \%$ ist vom Sturm geschädigt worden. Wenn die Kronenverlichtung von 1989 statt derjenigen von 1985 bekannt wäre, würde sich vermutlich ein noch engerer Zusammenhang mit den Sturmschäden 1990 crgeben. Da in diesem Forstbetrieb nur wenige Sturmschäden an Buchen festgestellt wurden, kann für diese Baumart nichts Entsprechendes ausgesagt werden.

Nachträglich ist dieser Zusammenhang auch für nationale Waldschadeninventur überprüft worden (SaNasilva 1991). Gesamtschweizerisch wurden in der Klasse mit 15 bis $25 \%$ Nadelverlust 2,5 mal so oft, in den Schadstufen mit mehx als $25 \%$ Nadelverlust sogar 3,5 mal so oft Bäume vom Sturm gefält wie in der Klasse mit 0 bis $10 \%$ Nadelverlust (Abb. 3). Die Häufigkeitsunterschiede würden vermutlich etwas korrigiert, wenn die gefällten Bäume auf alle Bäume im von den Stürmen 1990 heimgesuchten Gebiet statt auf diejenigen in der ganzen Schweiz bezogen werden könnten. Auf jeden Fall sind damit die lokal ermittelten Zusammenhänge für die ganze Schweiz klar bestärigt worden. 
* Sturmschaden 1990 / X storn damage 1990

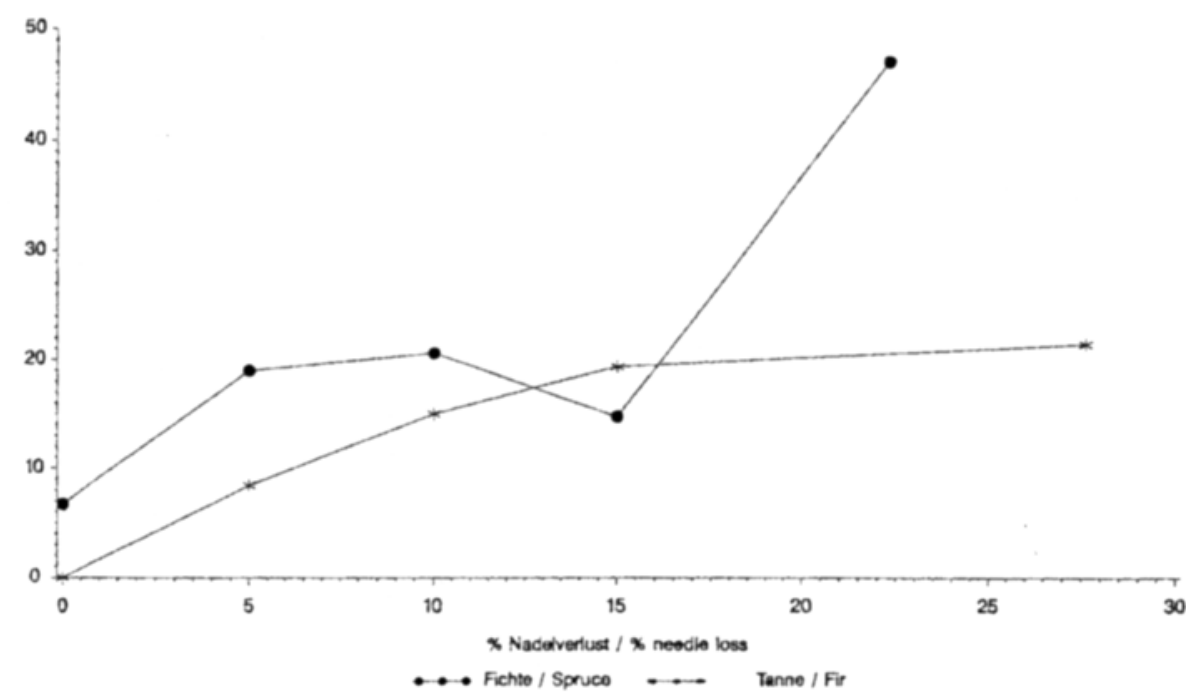

Arraly beobachtater Baume I Number of observed trees:

- 656

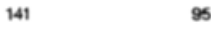

$95 \quad 17$

88

Abb. 2. Relative Häufigkeit der von den Winterstürmen 1990 in Oberentfelden gefällten Fichten und Tannen in Abbängigkeit vom Nadelverlust im Sommer 1985

Fig. 2. Relative frequency of Norway spruce and European white fir felled by storms in 1990, in relation to needle loss in 1985 (Community of Oberentfelden)

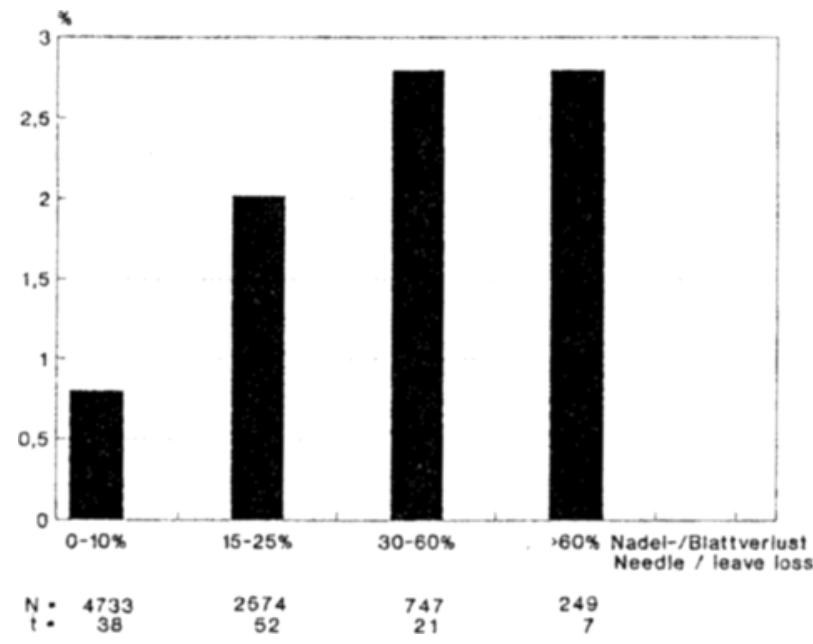

Abb. 3. Relative Häufigkeit der von den Winterstürmen 1990 in der Schweiz gefällten Bäume (alle Baumarten in Abhängigkeit rom Nadel-/Blattverlust im Sommer 1989 (Sanasilva 1991)

Fig. 3. Relative frequency of trees (all species) felled 1990 by storms in Switzerland, in relation to needle/leave loss in 1989 


\section{Folgerungen}

Der fehlende statistische Zusammenhang zwischen den nicht durch große Stürme bedingten Ausfallraten und den Bestandesaltern zwischen 80 und 210 Jahren, respektive den Stammdurchmessern in Plenterwald, zeigt, daß das beobachtete spontane Absterben und die anderweitig erzwungene Nutzung von Bäumen keine Alterserscheinungen sind, sondern meist auf schwere Schädigungen der betroffenen Individuen zurückgeführt werden müssen.

In der Schweiz kann ähnlich wie in Österreich nachgewiesen werden, daß die jährliche Sterberate bei schwacher Kronenverlichtung erst langsam, bei stärkerer Kronenverlichtung dann sehr rasch zunimmt. Kein einziges anderes Baum-, Bestandes- oder Standortsmerkmal konnte gefunden werden, das einen ähnlich guten Zusammenhang mit der Ausfallrate zeigt. Sowohl im Plenterwald wie auch im schlagweisen Hochwald kommt also den Nadel-/Blattverlusten für Fichten, Tannen und Buchen eine große prognostische Bedeutung zu.

Das besagt allerdings nicht, daß die Kronenverlichtung und somit der Mangel an assimilierenden Organen schon bei relativ geringen Nadel-/Blattverlusten cine erhöhte Sterblichkeit verursacht. Der statistische Zusammenhang ist einleuchtender dadurch zu erklären, daß auf eine bestimmte Art geschwächte Bäume bei Streß' einerseits etwas höhere Nadelverluste erleiden als andere und daß anderseits die Getahr ihres Ausscheidens größer ist (Abb. 4).

Der Abwurf alter und beschatterer Nadeln ist eine physiologisth sinnvolle Reaktion der Bäume, wcil damit die Transpiration eingeschränkt und anderweitig benötigte Nährstoffe zurückgewonnen werden. Wenn aber Bäume, die am wenigsten Nadeln verlieren, besser überleben als die andern, muß die Hypothese, nach welcher der vermehrte Abwurf von beschatteten und alien Nadeln cine gesunde Reaktion der viralsten Bäume bei gewissen Streßbelastungen darstelle, eindeutig verworfen werden. Dieser Hypothese widersprach schon, daß die Fichten und Tannen mit den geringsten Nadelverlusten meist seit längerer Zeit weitaus am besten gewachsen waren (SCHMid-HAAs 1990).

In mehreren Forstbetrieben konnce durch detaillierte Erhebungen und Analysen nachgewiesen werden, daß zumindest für Fichten und Tannen schon bei geringer Kronenverlichtung die Sturmgefährdung mit den Nadelverlusten statistisch signifikant zunimmt. Diese Beziehung ist für die Gesamtheit aller Baumarten in der ganzen Schweiz ähnlich.

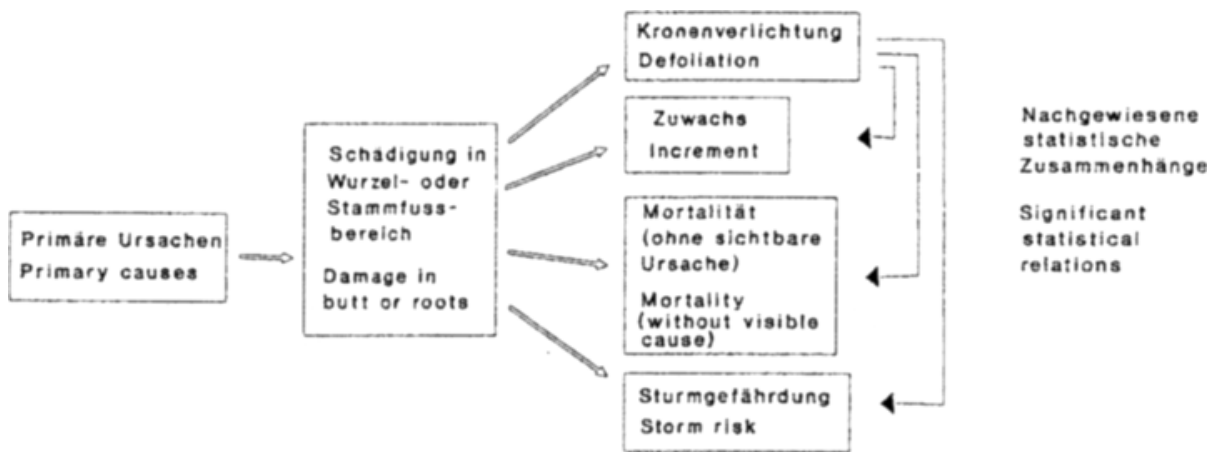

Abb. 4. Lrsächliche Zusammenhänge (Doppelpfeile), welche die ermittelten statistischen Beziehungen erklären können. Damit wird bewußt nur ein Teil der möglichen ursächlichen Zusammenhänge wiedergegeben; der Kausalnexus, das ursächliche Wirkungsgeflechr, kann in Wirklichkeit vicl komplexer sein

Fig. 4. Causal relations (double arrows) explaining the statistical correlations. Deliberately, the possible relations are described only partially; in reality, the causal nexus may be much more complex 
Der eindeutige statistische Zusammenhang zwischen Kronenverlichtung und Sturmgefährdung bei Tannen und Fichten kann natïrich nicht bedeuten, daß die fehlenden Nadeln direkt an der höheren Sturmanfälligkeit schuld sind. Lockere Kronen bieten dem Wind im Gegenteil weniger Widerstand und müssen daher einer kleineren Krafı widerstehen. Der statistische Zusammenhang kann überdies auch nicht dadurch erklärt werden, daß an Orten mit besonders großen Windstärken auch die Nadelverluste am höchsten seien. Eine (logistische) Kovarianzanalyse zeigt nämlich, daß der Zusammenhang zwischen Sturmgefährdung und Nadelverlust beim Vergleich der Bäume innerhalb der gleichen Probeflächen sogar größer ist als beim Vergleich der Bäume aus verschiedenen Probeflächen. Insbesondere nebeneinander stehende Bäume gleicher sozialer Stellung sind also verschieden stark gefährdet, wenn die Kronenverlichtung unterschiedlich ist.

Wenn Bäume mit leicht verlichteter Krone weit häufiger durch Stürme geschädigt werden als Bäume mit dichter Krone, kann das nur durch eine Schwächung im Wurzeloder unterem Stammbereich erklärt werden (SCHMLD-HAas 1991). Diese Schwäche muß so groß scin, daß die Minderbelastung der lichteren krone mehr als kompensiert wird. Die Art dieser Schwäche ist nicht bekannt. Irgendein Einfluß könnte bei einem Teil der Bäume zur Schwächung des Holzes, zu vermindertem Wurzelwachstum oder zu beschleunigtem Absterben der Feinwurzeln geführt haben.

Zwar ist bei mehr als $50 \%$ aller von den Stürmen cinzeln gebrochenen oder geworfenen Fichten, $13 \%$ der Tannen und $19 \%$ der Buchen Fäule an wichtigen Bruchstellen festgestellt worden (Schimd-HaAs u. Bachofen 1991). Die Sturmschäden wären ohne Fäuleeinfluß also wesentlich kleiner gewesen, der Anteil der Faule am gesamten Baumbestand bleibt aber unbekannt. Aus methodischen Gründen konnte in den bisherigen Untersuchungen auch nicht geklärt werden, ob die Fäulehäufigkeit mit den Nadelverlusten korreliert ist oder ob der statistische Zusammenhang zwischen Nadelverlusten und Sturmgefährdung durch eine andere Schwächung erklärt werden muß.

Die gleichgerichteten statistischen Zusammenhänge zwischen Nadetverlust und Sturmgetahrdung sowie zwischen Nadelverlust und Stcrberate ohne sichebare äußere Einwirkung legen nahe, daß auch die ursächlichen Zusammenhänge zum Teil ähnlich sein könnten. Eine Schwäche im Wurzel-oder unteren Stammbereich könnte auch die Sterbewahrscheinlichkeit ohne extreme Sturmeinwirkung erhöhen. Bei großen Nadelverlusten sind die statistischen Zusammenhänge mit der Sturmgefährdung und der übrigen Mortailtät verschieden, weil offenhar andere ursächliche Zusammenhänge wichtig werden.

Eine ähnliche Vermutung ist schon in bezug auf den engen statistischen Zusammenhang zwischen dem mitrleren Zurvachs eines Baumes in den letzten Jahrzehnten und seinem Nadelverlust geäußert worden. Eine Schwäche im Wurzel- oder Stammbereich, welche die Sterberate erhöht und den Nadelverlust vergrößert, könnte auch den Zuwachs reduzieren. Sotern diese Hypothese stimmt, müßte die Schwäche allerdings meist schon während 15 und mehr Jahren bestanden haben, da die Zuwachsunterschiede weit zurück reichen und oft keine rezenten Veränderungen des Zuwachses, die mit den Kronenverlichtungen im Zusammenhang stehen, nachweisbar sind.

Die Frage nach den primären Ursachen bleibt auch in diesem Fall offen. Sie muß aber neu formulier werden, weil die Frage, was die Schwäche im Stammfuß-oder Wurzelbereich verursacht habe, ein methodisch wesentich anderes Vorgehen bedingt als die Frage nach den direkten Ursachen der Kronenverlichtungen.

Die Ansprache der Nadel-/Blattverluste hat sich in den untersuchten Wäldern offenbar bewährt, denn die statistischen Zusammenhänge wären nicht erklärbar, wern dieses Merkmal nicht schon bei kleiner Kronenverlichtung relativ gut ertal3t worden wäre.

Von mehreren Autoren ist emptohlen worden, die Nadel-/Blattverluststufen $0-10 \%$ und $15-25 \%$ zuzammenzufassen, weil Bäume dieser Stufen noch keine Schädigung aufweisen sollen. Diese Begründung erschien schon bei Untersuchungen der Zuwächse zweifelhatt, weil Bäume dieser beiden Kronenverlichtungsstufen zwar ungefähr gleich 
häufig Zuwachseinbrüche gezeigt, sich in der absoluten Höhe des Zuwachses der letzten Jahrzehnte aber sehr deutlich unterschieden haben. Der statistisch signifikante Unterschied in einer so bedeutenden Größe wie der Sturmgefährdung zeigt nun eindeutig, daß diese beiden Kronenverlichtungsstufen nicht zusammengefaßt werden dürfen. Im Gegenteil sollten in möglichst viele Publikationen die Häufigkeiten in den $5 \%$-Klassen angegeben werden, in denen die Nadel-/Blattverluste richtigerweise meist sowieso erfaßit werden.

Die statistischen Zusammenhänge zwischen der Kronenverlichtung einerseits, jährlicher Ausfallrace, Sturmgefährdung und Zuwachs anderseits zeigen die große prognostische Bedeutung des Nadel-/Blattverlusts bei den untersuchten Baumarten. Nadel- und Blattverluste müssen daher unbedingt auch weiterhin bei jeder Waldschadeninventur angesprochen werden. Selbstverständlich soliten trotzdem auch andere Merkmale erprobt und bei Bewährung zusätzlich in die Waldschadeninventuren integriert werden.

Die Zunahme der Bäume mit mindestens $15 \%$ Nadel-/Blattverlust von $36 \%$ auf $68 \%$ aller Bäume der Schweiz von 1984 bis 1991 (Savasilva 1991), die bisher nur teilweise durch die Witterung erklärt werden kann, muß um so mehr beunruhigen, als der Kronenverlichtung eine große Bedeutung nicht nur für die langfristige Prognose des Zuwachses, sondern auch für die Sturmgefährdung und die Ausfallrate ohne extremen Sturmeinfluß zukommr. Die zunehmende Kronenverlichtung scheint ein erhöhtes Risiko für den Wald zu signalisieren.

\section{Zusammenfassung}

In Plenterwäldern und in gleichaltrigen, regelmäißig geptlegten Waldbeständen in Mittelland, Voralpen, Jura und Alpen wurden die Kronen von Fichten, Tannen und Buchen angesprochen und die Bäume dann während mehrerer Jahre auf ihr Überleben oder Ausscheiden beobachet.

Bei Fichten, Tannen und Buchen sind die jährlichen Ausfallraten mit den angesprochenen Kronenverlichtungen korreliert. Der Einfuß der Kronenverlichtung auf die Ausfallraten ist für alle drei Baumarten statistisch signifikanr. Keines der anderen angesprochenen Srandorts-, Bestandes- und Einzelbaummerkmale trägt zusätzlich zur Schätzung der Ausfallrate bei.

Auch die Fichten und Tannen, die von den Winterstürmen 1990 gefällt worden sind, haben signifikant größere Nadelverluste aufgewiesen als die stehengebliebenen. Die durch Stürme bedingten Sterberaten sind schon bei $10 \%$ Nadelverlust bedeutend größer als bei $0 \%$.

Die engen statistischen Zusammenhänge zwischen der Kronenverlichtung, der Ausfallrate ohne extremen Sturmeinfluß und der Sturmgefährdung zeigen die große Bedeurung des Nadel-/Blattverlusts für die Prognose des Überlebens oder Ausscheidens vor allem bei Fichten und Tannen.

\section{Summary \\ Crown transparency and mortality rates in sprace, fir and beech}

The crowns of spruces, firs, and beeches in selection forests and evenaged regularly managed stands in the Swiss Mirrelland, the Pre-Alps, the Jura and the Alps were assessed, and the trees subsequently observed over several years in terms of survival or mortality.

The mortality rate was found to be statisticaily significant in relation to crown transparency. There was no correlation indicating additional effects of other parmeters estimaced, i.e. site condicions, stand conditions, or features of individual trees.

Spruce and firs blown by the winter storms of 1990 displayed a significantly greater needle loss than those which survived. Mortality rates due to storm damage were already considerably greater in trees with 10 percent needle loss than in those with 0 percent needle loss.

The close statistical relationships between crown transparency, mortality rates in the abscnce of extreme storms, and storm hazard show the great significance of leaf or needle loss for the survival or death of trees, especially for fir and spruce.

\section{Literatur}

DONG, P. H.; Kramer, H., 1987: Zuwachsverlust in erkrankten Fichtenbeständen. Allg. Forst- u. J.-Zcg. 158, 122-125.

Fischer, U.; ReIrer, C., 1985: Waldschadeninventur, OBG Oberentfelden 1985. Vervielfalltigung. 
KeLleR, W.; IMHOF, P., 1987: Zum Einfluß der Durchforstung auf die Waldschäden. Schweiz. Z. Forstwes. 138, 39-54 und 293-320.

KELLER, W.; STARK, M., 1991: Wachstum und Kronenverlichtung auf den Beobachtungsflächen Lägeren, Alptal und Davos. In: Luftschadstoffe und Wald, 31-36. Verlag der Fachvereine an den schweizerischen Hochschulen und Techniken, Zürich.

LÄTT, N., 1991: Zum Zusammenhang zwischen Kronenschäden und Windfallholzanteil. Schweiz. Z. Forstwes. 142, 109-131.

LESINSKi, J. A.; LANDMANN, G., 1988: Crown and Branch Malformation in Conifers Related to Forest Decline. In: Scientific Basis of Forest Decline Symptomatology (Ed. J. N. Cape, P. Mathy), 92-105. Air Pollution Report Series, No. 15.

Sanasilva-Waldschadenbericht 1991. BUWAL, Bern und Eidg. Forschungsanstalt für Wald, Schnce und Landschafr, Birmensdorf.

Schmid-HaAs, P.; 1990: Kronenverlicheung und Waldwachstum. Schweiz. Z. Forstwes. 141, 189-209.

- 1991: Ursächliche Zusammenhänge zwischen Nadelverlust, Zuwachs, Sturmgetährdung und Fäule. Schweiz. Z. Forstwes. 142, 505-512.

SCHMid-HaAs, P.; BaChofEN, H. 1991: Die Sturmgetährdung von Einzelbäumen und Beständen. Schwciz. Z. Forstwes. 142, 477- 504.

Stemberger, A. 1991: Über Ausmaß und Verteilung der Mortalität: Gegenüberstellung von Ergebnissen der Waldzustandsinventur mit früheren Untersuchungen. Mitceilungen forstl. Bundesversuchsanstalt Wien, 166, 163-173.

Auschrift des Verfassers: Prof. Dr. Paul Scumid-Haas, Eidgenössische Forschungsanstalt für Wald, Schnee und Landschaft, Zürcherstraßje 11, CH-8903 Birmensdorf 